\title{
Analysis of the attacker and defender GAN models for the indoor navigation network
}

\author{
L. Niu ${ }^{1, *}$, Y. Song ${ }^{2}$, J. Chu ${ }^{1}$, S. Li ${ }^{1}$ \\ ${ }^{1}$ School of Geomatics, 730072, Anningxilu, Lanzhou, China, 1.niu@mail.lzjtu.cn \\ ${ }^{2}$ School of Geographic and Environmental Science, Normal University of Tianjin, West Bin Shui Avenue, 300387 Tianjin, China - \\ syiq@hotmail.com
}

\section{Commission IV}

KEY WORDS: Deep learning, Generative-Adversarial-Neural network, attacker, defender, indoor navigation network

\begin{abstract}
Evacuation research relies heavily on the efficiency analysis of the study navigation networks, and this principle also applies to indoor scenarios. One crucial type of these scenarios is the attacker and defender topic, which discusses the paralyzing and recovering operations for a specific indoor navigation network. Our approach is to apply the Generative-Adversarial-Neural network (GAN) model to optimize both reduction and increase operations for a specific indoor navigation network. In other words, the proposed model utilizes GAN both in the attacking behavior efficiency analysis and the recovering behavior efficiency analysis. To this purpose, we design a black box of training the generative model and adversarial model to construct the hidden neural networks to mimic the human selection of choosing the critical nodes in the studying navigation networks. The experiment shows that the proposed model could alleviate the selection of nodes that significantly influence network transportation efficiency. Therefore, we could apply this model to disaster responding scenarios like fire evacuation and communication network recovery operations.
\end{abstract}

\section{INTRODUCTION}

\subsection{A general introduction of deep learning models}

The indoor navigation network is crucial for many application scenes like indoor routing and emergency evacuation, and the research results from these studies could significantly reduce the total evacuation time and egress routes' length (Scholz and Schabus, 2014, Zlatanova et al., 2013, Liu and Zlatanova, 2011, Krūminaitè and Zlatanova, 2014, Gozick et al., 2011, KirkkoJaakkola et al., 2013). Therefore, many researchers committed contributions to investigate the robustness for this specific type of navigation networks (Freitas and Chau, 2020, Alattas et al., 2017, Fellner et al., 2017, Snelder et al., 2012, Santos et al., 2010).

The graphs studies usually are in the form of networks, which are widely used for processing several critical graphs and applications like transportation network analysis and a significant loss. This approach originated from the graph convolution network, which uses deep networks to extract global and local graphs. The specific graphs for this approach are widely used along with this approach and promising for many fields. They can still conclude several layers of deep learning networks, consisting of millions of parameters for a specific model. To mitigate the specific challenges brought by the indoor navigation environment, we have to tune these models for our specific usage.

Fortunately, we could refer to many existing methods that could efficiently handle the challenges of introducing deep learning techniques for indoor navigation networks. One of the most heavily studied solutions is based on the generative adversarial network (GAN) framework (Liu et al., 2019, Suárez et al., 2017, Martin Arjovsky, 2017, Qiyue Li 2020, Jonas Adler 2018, Cao et al., 2019). Thus, we follow this approach due to its advantages in taking both the global and local processing information features.

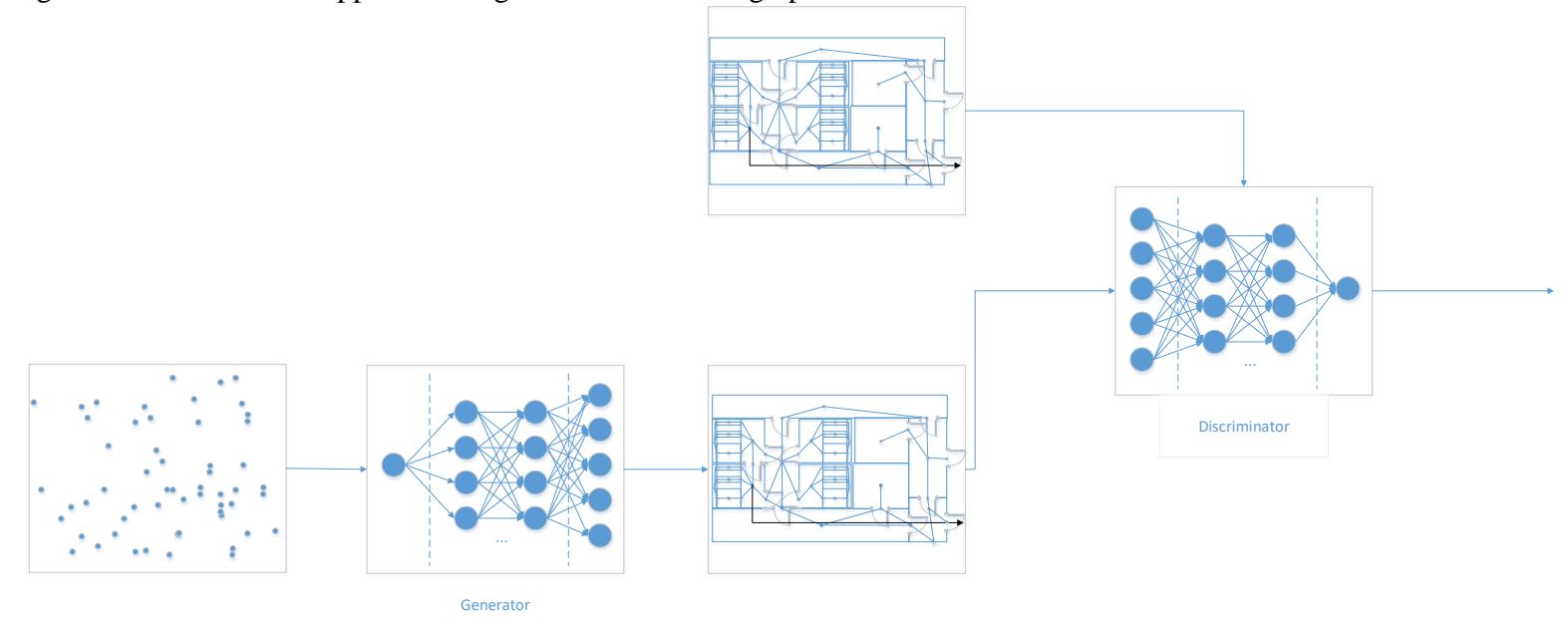

Figure 1. The demonstration of the GraphGAN for our approach

\footnotetext{
* Corresponding author
} 
Along with our approach, we utilize the GAN that uses a generator and discriminator to study the connection features of the navigation graphs in figure 1 (Hou et al., 2019, Suárez et al., 2017, Adler and Lunz, 2018). Although our current investigation solution is premature and naïve, we still believe it could extract critical features from the whole navigation network that will influence the network's connectivity status. As a GAN framework, one part of this model is named the 'generator'; the other network is named the 'discriminator.' The first one that is in charge of generating objects as realistic as the studying examples; while the latter one act like judges, who will distinguish the fake pictures of networks, which are previously generated by the generator. Using the generator and discriminator, we can identify fake objects like pictures that are very like the actual pictures generated by a selected optimizer. For our purpose, we use the generator and discriminator function as the attacking and defending of the critical nodes of the navigation networks (Freitas and Chau, 2020, Duan and Lu, 2014, Zlatanova et al., 2013, Liu and Zlatanova, 2011, Krūminaitè and Zlatanova, 2014).

\section{THE MECHANISM OF GRAPHGAN FOR INDOOR NAVIGATION NETWORK ANALYSIS}

We build this model because we are inspired by the GraphGAN project from GitHub: https://github.com/hwwang55/GraphGAN/tree/3f1c3f7db6b8b5 102 eea899659d4c52f64c4288c, which is shown in figure 2 . The composition mechanism is quite straight, and we inherit the main schema of this GraphGAN framework. Thus, we establish the generator with a softmax mechanism to minimize the logarithm result between the produced graphs and the learning graph layers. On the contrary, the introduced discriminator aims to maximize the logarithm result between the provided graph results mixed with the original graphs and the generatorproduced graphs. The detail foundation theory and proving detail could be found in the original GraphGAN paper and is generally demonstrated in figure 2 .

The detail softmax mechanism is a graph softmax operation. This graph softmax is implemented by the Adam optimizer both across the generator and discriminator. Besides, there are three modes for the GraphGAN operations: link-prediction, node classification and recommendation. The first one is the process of generating fake edge by the generator and identification by the discriminator; while the second one is the process of generating different groups of edges that have labels to distinguish them; and the last one is to utilize the connectivity relationship to find recommendations between different nodes. We currently only consider the former two types of operations.

The detail operation flow is:

1. Use the word $2 \mathrm{vec}$ libraries to embed the comparative similarity rate between different graph structures;

2. Then use the previously produced embedding matrix to compute the generated graph edges for link prediction and node groups;

3. Generate the output matrixes by executing the Tensorflow loops.

Figure 2 shows that the working performance of the generator. As in the left part of this figure, we could easily perceive that the accessible nodes selected by the generator does not overlap with any of the existing target learning nodes. Thus, under this circumstance, the generator fails to achieve the link generation purpose, and we name this situation as the underperformance. While for the central part, the generated nodes set is partially overlapping the existing target node set, and we name this situation as the approaching truth situation. For the last part of the figure, due to the selection set of the generator is nearly overlapping with the whole truth nodes set, thus the performance of using adversial training for generator is achieved.

The most critical settings of Tensorflow based GraphGAN structures are the outer iterations and inner iterations for the computing process because these settings determine the studying result of the GraphGAN softmax layers on the input graph connection matrixes.

Following this approach, without drowning with the tedious work of tuning hyper-parameter for our model, we can concentrate on optimizing the mechanism of generator and discriminator for identifying the indoor navigation networks.
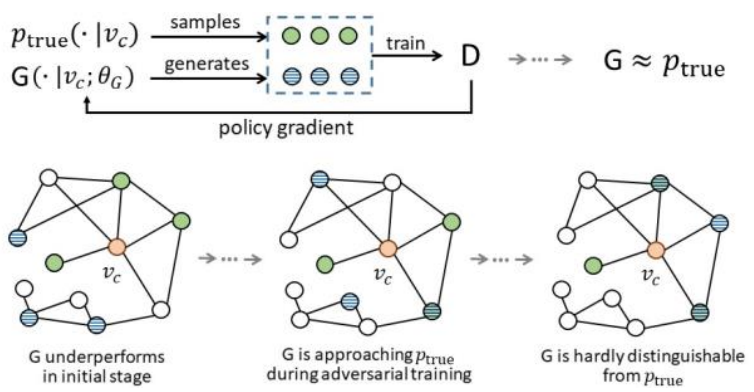

Figure 2. The mechanism of the GraphGAN (by courtesy of Hongwei Wang, etc..)

These random indoor navigation networks that mainly consist of one hundred nodes. These networks have a maximum of four degrees of connectivity for the nodes in them. Although this setting connectivity degree is not thoroughly studied through the theoretical research, we extract this setting from a complete survey from the observation of realistic buildings from both the Henan university of urban construction number 5 building and the Meiluocheng building shanghai city in figure 3 and 4 .

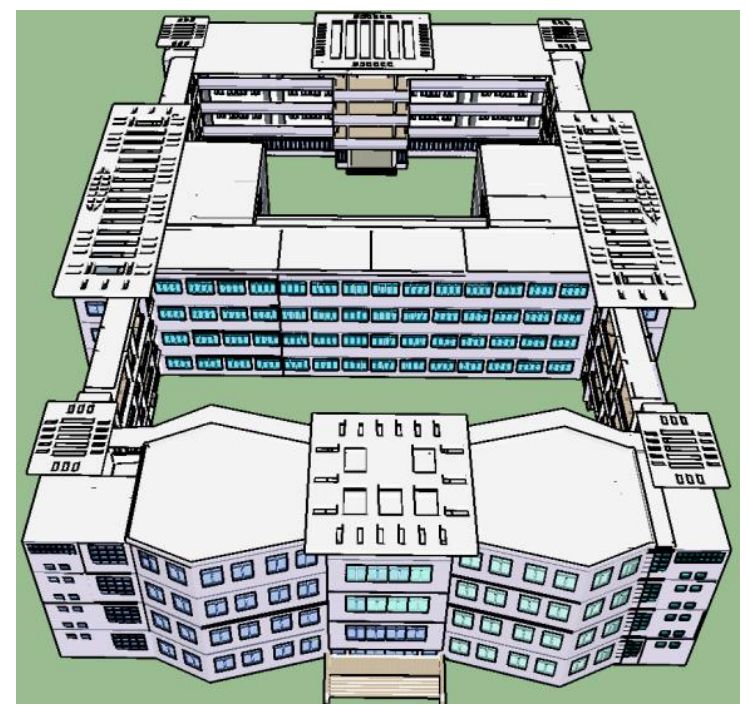

Figure 3. The overview of the Henan University of Urban Construction Number 5 building 


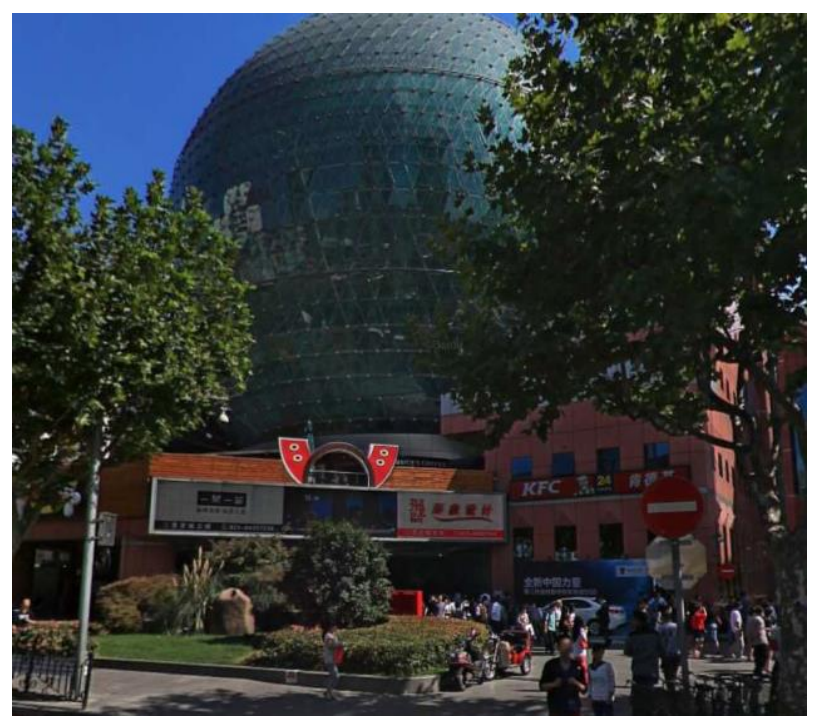

Figure 4. The overview of the Meiluocheng building

\section{DATASET AND EXPERIMENT RESULTS}

\subsection{Test platform and configurations}

Test platform hardware configuration is 16 core Ryzen $93950 \mathrm{X}$ with 64GB DDR3000 rams and Geforce GTX 2070; the software platform is Ubuntu 20.04 LTS; the deep learning framework is Tensorflow 2.2 GPU. The detail configuration setting of the GraphGAN model is listed below:

\author{
\# training settings \\ batch_size_gen $=64$ \\ batch_size_dis $=64$ \\ lambda_gen $=1 \mathrm{e}-5$ \\ lambda_dis $=1 \mathrm{e}-5$ \\ n_sample_gen $=20$ \\ lr_gen $=1 \mathrm{e}-3$ \\ lr_dis $=1 \mathrm{e}-3$ \\ n_epochs $=20$ \\ n_epochs_gen $=30$ \\ n_epochs_dis $=30$ \\ gen_interval $=$ n_epochs_gen \\ dis_interval $=$ n_epochs_dis \\ update_ratio $=1$ \\ \# model saving \\ load_model $=$ False \\ save_steps $=10$ \\ \# other hyper-parameters \\ n_emb $=128$ \\ window_size $=2$ \\ multi_processing $=$ False
}

'batch_size_gen' parameter determines the batch size for the generator; 'batch_size_dis' parameter determines the batch size for the discriminator; 'lambda_gen' parameter determines the 12 loss regulation weight for the generator; 'lambda_dis' parameter determines the 12 loss regulation weight for the discriminator; ' $n$ _sample_gen' parameter determines the number of samples for the generator; 'lr_gen' parameter determines the learning rate for the generator; 'Ir_dis' parameter determines the learning rate for the discriminator; 'n_epochs' parameter determines the number of outer loops; 'n_epochs_gen' parameter determines the number of inner loops for the generator; 'n_epochs_dis' parameter determines the number of inner loops for the discriminator; 'gen_interval' parameter determines the sample new nodes for the generator for every generator interval iterations; 'dis_interval' parameter determines the sample new nodes for the discriminator for every discriminator interval iterations; 'update_ratio' parameter determines the updating ratio when choose the trees; 'load_model' parameter determines whether loading existing model for initialization; 'save_steps' parameter determines the step saving frequencies; ' $\mathrm{n}$-emb' parameter determines the embedding matrix's mapping node number; 'window_size' parameters determine the searching windows size; 'multi_processing' parameter determines whether using multi-processing to construct BFS-trees. The BFS-tree here means the breadth first searching tree formed for the specific node.

The experiments are executed on these random networks set with 100 nodes and 4 degrees of the maximum connection degree. We choose these specific node numbers and degree settings because, according to our current study of the existing floor, blueprints of several collected buildings are with these settings.

Because the research of GraphGAN on these navigation networks is scarce and rare, we can only extract several key features favoured from our aspects. A complete, more objective refinery of the raw data from the defined GAN produced results is expected in the following research step.

\subsection{Experiment result types}

The first result is named the uniform manifold approximation and projection (UMAP); the second result is the 'Student- $t$ distribution stochastic neighbor embedding' ( $t$-SNE in short); the third result is the principal component analysis (PCA) analysis result. The first two types of results show the analyzing data correlations across high dimensional space, and the last one shows the traditional correlations between any combination of selected features.

\section{DISCUSSIONS AND EXTENSIVE STUDY OF RESULTS}

From the results, we can find that these two networks are producing quite complex results, and the reason we have not provided the loss results of the experiment is due to that the current stage of our research is to testify the working flow of the combination between GraphGAN and the indoor navigation networks, we only committed the loops with the minimum setting of the GraphGAN model. This minimum setting is of 10 epochs for the input simulated graphs, thus the loss results of this simulation mechanism could not provide a complete overview of the total learning performance for this model. Besides, because we have not thoroughly investigated the different setting of the GraphGAN model.

The PCA results demonstrate the different mapping between the generated indoor navigation networks embedding relationships across different groups. The results provided below are these results among classes 1,2 , and $3 ; 4,5$, and $6 ; 7,8$, and 9, shown in Figures 5, 6, and 7.

The UMAP results show the graphs have relatively sparse distributions of the network: most of the nodes are distributed along a centric area but without prominent spatial distributions. The results provided are the snapshots of using 5 neighbors, 25 
neighbors, and 50 neighbors for this type of data evaluation aspect in Figures 8, 9, and 10.

The analysis result for t-SNE shows step 1003 for the iterations of the Student-t distribution stochastic neighbor embedding features for the iteration between all evaluation nodes in the test graphs, shown in figure 11.

Figure 5. The demonstration of the PCA 1-2-3 result.

Figure 6. The demonstration of the PCA 4-5-6 result.

Figure 7. The demonstration of the PCA 7-8-9 result.

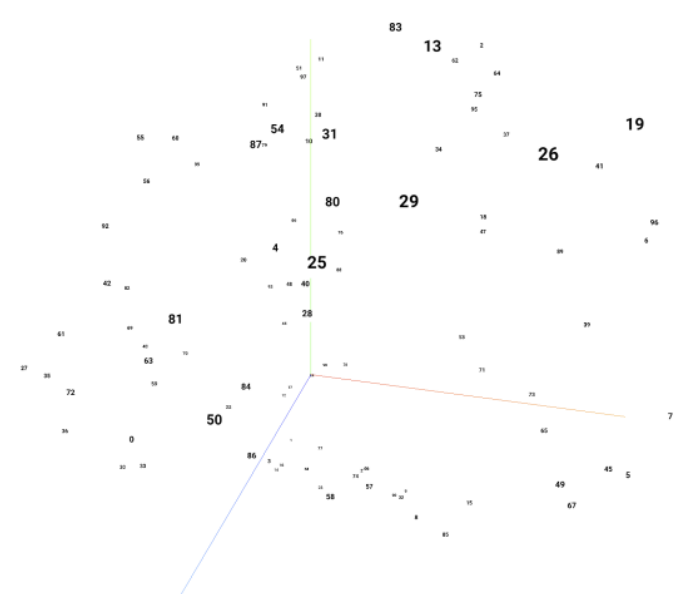

Figure 8. The demonstration of the UMAP 5 neighbors' result.

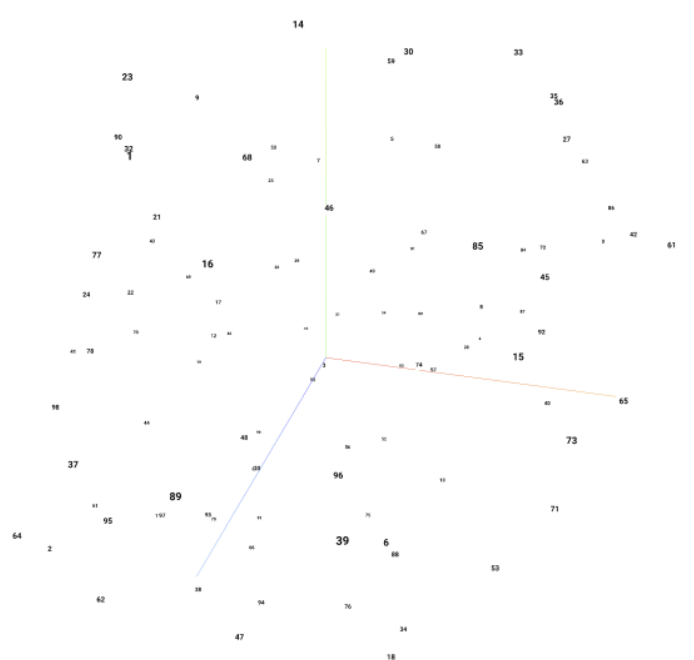

Figure 9. The demonstration of the UMAP 25 neighbors' result.

Figure 10. The demonstration of the UMAP 50 neighbors' result. 


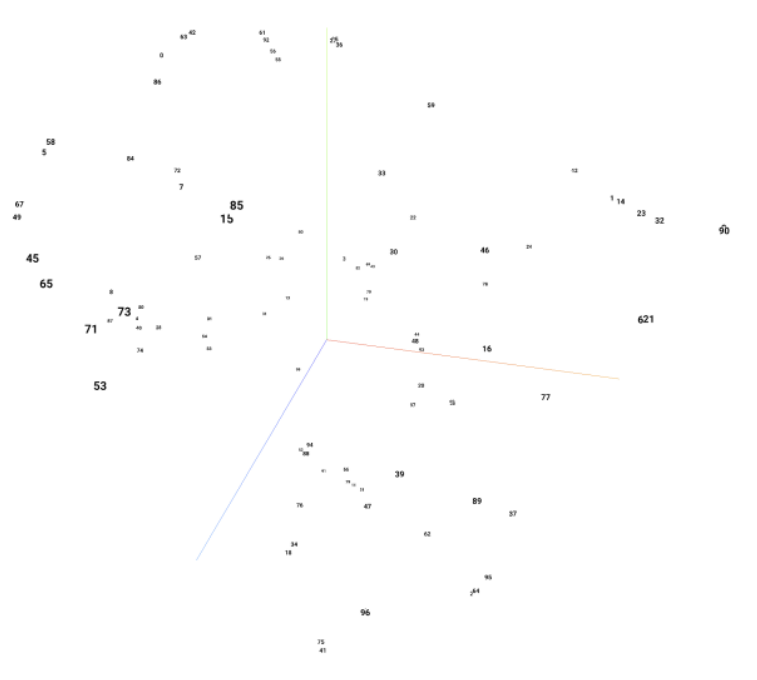

Figure 11. The demonstration of the t-SNE result with 1003 steps of iterations.

\section{CONCLUSIONS}

Aiming to solve the graph analysis problems by introducing GAN models, we have successfully established a foundational GraphGAN framework for analyzing the critical nodes in indoor navigation works. Although the current solution is naïve and preliminary, it still proposes a promising solution for starting the first step to study more complex and large-scale indoor navigation networks.

To mitigate the current bottlenecks of the proposed solution, we plan to configure the hyper test parameters with more loops, which may exceed 10,000 loops. An extensive set of nodes may exceed 10,000 for the number of nodes and connection degree more significant than 10 . However, we still have to pay enough attention to the balance between payouts of data model analysis and efficiency without introducing an overwhelming computational burden on test platforms with acceptable price.

\section{ACKNOWLEDGEMENTS}

This paper is sponsored by the National Science Foundation of China (41771433, 41501440, 41571387, 41471341); Natural Science Foundation of Gansu Province (20JR10RA247); Tianjin Natural Science Foundation (No.18JCYBJC90600).

\section{REFERENCES}

ADLER, J. \& LUNZ, S., 2018. Banach wasserstein gan. arXiv preprint arXiv:1806.06621,

ALATTAS, A., ZLATANOVA, S., VAN OOSTEROM, P. CHATZINIKOLAOU, E., LEMMEN, C. \& LI, K.-J., 2017. Supporting Indoor Navigation Using Access Rights to Spaces Based on Combined Use of IndoorGML and LADM Models. ISPRS international journal of geo-information, 6(12), 384,

CAO, J., MO, L., ZHANG, Y., JIA, K., SHEN, C. \& TAN, M., 2019. Multimarginal wasserstein gan. arXiv preprint arXiv:1911.00888,

DUAN, Y. \& LU, F., 2013. Structural robustness of city road networks based on community. Computers, Environment and Urban Systems, 41(75-87,

DUAN, Y. \& LU, F., 2014. Robustness of city road networks at different granularities. Physica A: Statistical Mechanics and its Applications, 411(21-34,
FELLNER, I., HUANG, H. \& GARTNER, G., 2017. "Turn Left after the WC, and Use the Lift to Go to the 2nd Floor"-Generation of Landmark-Based Route Instructions for Indoor Navigation. ISPRS International Journal of Geo-Information, 6(6), 183,

FREITAS, S. \& CHAU, D. H., 2020. Evaluating Graph Vulnerability and Robustness using TIGER. arXiv preprint arXiv:2006.05648,

GOZICK, B., SUBBU, K. P., DANTU, R. \& MAESHIRO, T., 2011. Magnetic maps for indoor navigation. Instrumentation and Measurement, IEEE Transactions on, 60(12), 3883-3891,

HOU, S., FAN, Y., ZHANG, Y., YE, Y., LEI, J., WAN, W., WANG, J., XIONG, Q. \& SHAO, F., 2019. $\alpha$ Cyber

: Enhancing Robustness of Android Malware Detection System against Adversarial Attacks on Heterogeneous Graph based Model. Proceedings of the 28th ACM International Conference on Information and Knowledge Management. $10.1145 / 3357384.3357875$.

ISIKDAG, U., ZLATANOVA, S. \& UNDERWOOD, J., 2013. A BIMOriented Model for supporting indoor navigation requirements. Computers, Environment and Urban Systems, 41(112-123,

JONAS ADLER , S. L., 2018. Banach Wasserstein GAN. 32nd Conference on Neural Information Processing Systems. Montréal.

KIRKKO-JAAKKOLA, M., COLLIN, J. \& TAKALA, J., Using Building Plans and Self-Contained Sensors with GNSS Initialization for Indoor Navigation. Vehicular Technology Conference (VTC Spring), 2013 IEEE 77th, 2013. IEEE, 1-5,

KRŪMINAITÉ, M. \& ZLATANOVA, S., Indoor space subdivision for indoor navigation. the Sixth ACM SIGSPATIAL International Workshop on Indoor Spatial Awareness, 2014 Dallas, Texas, USA. ACM, 25-31

LIU, H., GU, X. \& SAMARAS, D., Wasserstein gan with quadratic transport cost. Proceedings of the IEEE/CVF International Conference on Computer Vision, 2019. 4832-4841,

LIU, L. \& ZLATANOVA, S., 2011. Towards a 3D network model for indoor navigation. Urban and Regional Data Management, UDMS Annual, 79-92,

LIU, L. \& ZLATANOVA, S., A semantic data model for indoor navigation. the Fourth ACM SIGSPATIAL International Workshop on Indoor Spatial Awareness, 2012. ACM, 1-8,

MARTIN ARJOVSKY, S. C., AND LEON BOTTOU. 2017. Wasserstein GAN.

NIU, L. \& SONG, Y., 2019. A Faster R-CNN Approach for Extracting Indoor Navigation Graph from Building Designs. International Archives of the Photogrammetry, Remote Sensing and Spatial Information Sciences, 42(2/W13),

OZDENIZCI, B., OK, K., COSKUN, V. \& AYDIN, M. N., Development of an indoor navigation system using NFC technology. Information and Computing (ICIC), 2011 Fourth International Conference on, 2011. IEEE, 11-14,

QIYUE LI , M., IEEE,HENG QU,ZHI LIU , MEMBER, IEEE, NANA ZHOU, WEI SUN, STEPHAN SIGG, MEMBER, IEEE,AND JIE LI, 2020. AF-DCGAN: Amplitude Feature Deep Convolutional GAN for Fingerprint Construction in Indoor Localization Systems. IEEE Transactions on Emerging Topics in Computational Intelligence, 1-13, 10.1109/tetci.2019.2948058.

SANTOS, B. F., ANTUNES, A. P. \& MILLER, E. J., 2010. Interurban road network planning model with accessibility and robustness objectives. Transportation Planning and Technology, 33(3), 297-313,

SCHOLZ, J. \& SCHABUS, S., 2014. An indoor navigation ontology for production assets in a production environment. Geographic Information Science. Heidelberg, Germany: Springer,

SNELDER, M., VAN ZUYLEN, H. \& IMMERS, L., 2012. A framework for robustness analysis of road networks for short term variations in supply. Transportation Research Part A: Policy and Practice, 46(5), 828-842,

STOOK, J., 2011. Planning an indoor navigation service for a smartphone with Wi-Fi fingerprinting localization. Master thesis, TU Delft, The Netherlands.

SUáREZ, P. L., SAPPA, A. D. \& VINTIMILLA, B. X., Infrared image colorization based on a triplet dcgan architecture. Proceedings of the IEEE Conference on Computer Vision and Pattern Recognition Workshops, 2017. 18-23,

SUN, Y., DING, N., QIAN, H. \& XU, Y., Real-time monocular visual selflocalization approach using natural circular landmarks for indoor navigation. 2012 IEEE International Conference on Robotics and Biomimetics (ROBIO), 2012. IEEE, 495-500, 
XU, W., KRUMINAITE, M., ONRUST, B., LIU, H., XIONG, Q. \& ZLATANOVA, S., 2013. A 3D Model Based Indoor Navigation System for Hubei Provincial Museum. ISPRS-International Archives of the Photogrammetry, Remote Sensing and Spatial Information Sciences, 1(4), 51-55,

YUAN, W. \& SCHNEIDER, M., 2010. iNav: An Indoor Navigation Model Supporting Length-Dependent Optimal Routing. Geospatial Thinking, 299-313,

ZLATANOVA, S., LIU, L. \& SITHOLE, G., A conceptual framework of space subdivision for indoor navigation. Proceedings of the Fifth ACM SIGSPATIAL International Workshop on Indoor Spatial Awareness, 2013. ACM, 37-41, 